\title{
Effectiveness of Periarticular Infiltration of the Knee during Total Knee Arthroplasty for Postoperative Pain Management
}

\author{
Bibhuti Nath Mishra, ${ }^{* 1}$ Joydeep Banerjee Chowdhury, ${ }^{2}$ Rajeev Raman, ${ }^{2}$ Dipmalya Chakraborty, ${ }^{3}$ \\ Tanmoy Karmokar ${ }^{3}$
}

\author{
Author Info: \\ ${ }^{1}$ Lecturer, Birat Medical College \\ Teaching Hospital, Tankisinwari, \\ Biratnagar, Nepal \\ ${ }^{2}$ Consultant Orthopaedic \\ Surgeon, Joint \& Bone Care \\ Hospital, Salt Lake City, Kolkata, \\ India \\ ${ }^{3}$ Orthopaedic Registrar, Joint \& \\ Bone Care Hospital, Salt Lake \\ City, Kolkata, India
}

\section{*Corresponding Author:}

Dr. Bibhuti Nath Mishra, Department of Orthopaedics, Birat Medical College Teaching Hospital, Tankisinwari, Biratnagar, Nepal.

Email/Contact: drbibhuti5@ gmail.com, Phone: +9779841216778

\begin{abstract}
Background: Different treatment regimens of analgesia, nerve blocks, and epidurals are used for pain relief in Total Knee Arthroplasty (TKA). Local infiltration analgesia (LIA) is one of the modalities in which a cocktail combination of different medicines is infiltrated locally into the capsule, surrounding tissues, or intraarticular joint space. This study aims to analyze the effectiveness of periarticular injection of combination drugs (Bupivacaine, Ketorolac, and Morphine) during TKA for postoperative pain management.
\end{abstract}

Methods: This was an experimental comparative study. A total of 150 patients who underwent primary unilateral TKA were randomly categorized into 2 groups (75 each). Group-A (control group) didn't receive intraoperative periarticular injection and Group-B received the intraoperative injection of combined local analgesics and anesthetics (Bupivacaine, Ketorolac, and Morphine). Pain following surgery at 0, 1, and 2 postoperative days was recorded with visual analogue scale (VAS) whereas Knee Society Score was used to evaluate the pain and function pre-operative and 3 months post-operative. Statistical analysis was done using IBM SPSS software version 16.

Results: Patients receiving periarticular infiltration of combination drugs intraoperatively had lower VAS for postoperative pain $(p<0.001)$ and this group also showed the reduced need for analgesia postoperatively.

Conclusions: Periarticular infiltration of the knee during Total Knee Arthroplasty is effective in the management of postoperative pain

Keywords: Humans; Replacement; Arthroplasty; Knee

QR Code
Received: 19 June 2021; Article Info
How to cite this article in Vancouver Style?




\section{INTRODUCTION}

The fear of post-operative pain in an elective procedure like Total Knee Arthroplasty (TKA) remains a big concern to patients making it an underused surgery. ${ }^{1}$ Pain threshold differs among individuals but severe post-operative pain affecting rehabilitation is reported in $50 \%$ of TKA patients. ${ }^{2}$ The role of adequate analgesia and anesthesia during primary TKA is discussed in literature ${ }^{3}$ and different treatment regimens of analgesia, nerve blocks, and epidurals are mentioned. ${ }^{4,5}$ Local infiltration analgesia (LIA) is one modality with a cocktail combination of different medicines infiltrated locally into the joint., 4 But its superiority to other modalities is still not established. Though epidural analgesia has proven benefits, its multiple side effects make it a little risky choice. ${ }^{6-8}$ Thus, the use of local combinations of analgesics at the surgical site with minimum side effects is a good choice. ${ }^{9}$ This study aims to analyze the effectiveness of periarticular injection of combination drugs (Bupivacaine, Ketorolac, and Morphine) during TKA for postoperative pain management.

\section{MATERIALS AND METHODS}

This was an experimental comparative study conducted from January 2019 to December 2019. The study was conducted at Joint \& Bone Care Hospital, Salt Lake City, Kolkata, India and all cases were operated by a single surgeon. Ethical Clearance was taken from the institutional review committee of same institution (Ref. No. JBC/0001/19-20) before starting the study and informed consent was taken from all patients. All cases of painful osteoarthritic (degenerative/non-inflammatory) knee joints planned for primary unilateral TKA in specified study duration were included in this study. Elderly patients with hypertension and diabetes control with routine use of medicines were included. Patients with cardiac comorbidities, chronic kidney or liver disease, and allergic to drugs used in this study were excluded. Pre-operative evaluation and documentation of pain were done using the Visual analogue scale (VAS).

The patients were randomly assigned to Group-A and Group-B on the alternate basis of odd and even number. One group (Group-A) didn't receive the intraoperative periarticular injection and another (Group-B) received the intraoperative injection of combined local analgesics and anesthetics. All surgeries were performed under spinal anesthesia and those cases in which the modality of anesthesia has to be changed to GA intraoperatively were discarded from this study. The other intraoperative and postoperative anesthetic regimes were the same for both groups. Two vials
( $20 \mathrm{ml}$ each) of $0.25 \%$ Bupivacaine Hydrochloride Injection $(2.5 \mathrm{mg} / 8 \mathrm{mg} / 1 \mathrm{ml}), 2$ ampoules of ketorolac Tromethamine Injection $(30 \mathrm{mg} / \mathrm{ml})$, and 1 ampoule of Morphine Sulphate Injection $(15 \mathrm{mg} / \mathrm{ml}$ ) were uploaded in a syringe of $50 \mathrm{ml}$ (Figure 1). It was not diluted with Normal Saline or any other drugs. After completing the femoral and tibial bone cuts and just before placement of implants, these combination drugs were infiltrated using 20 gauge 1 and $1 / 2$ inches long needles and targeting the 8 anatomical zones ${ }^{10}$ of the knee with abundant sensory innervations (Figure 2). While infiltrating the posterior capsule (medially and laterally), care was taken to avoid infiltration into the popliteal artery. Pain following surgery at day 0 , 1st, 2nd, and 3rd postoperative days were recorded with VAS $(0=$ No pain at all; $10=$ maximum pain

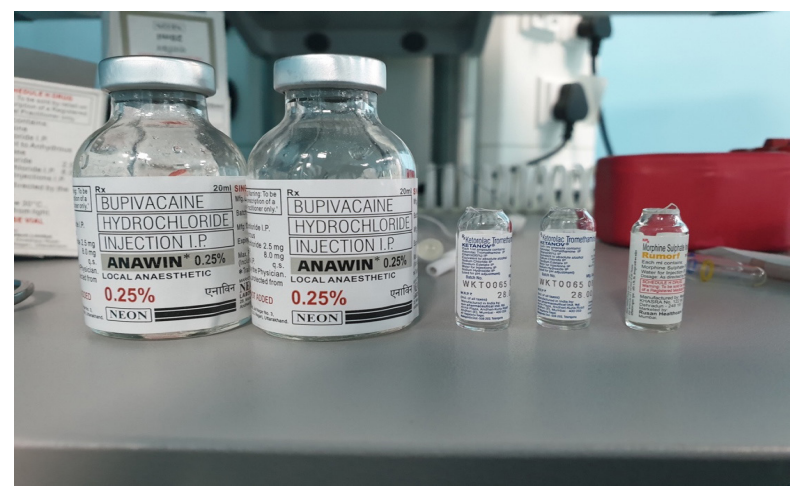

Figure 1: Drugs used in "cocktail" combination of periarticular Injection of TKA

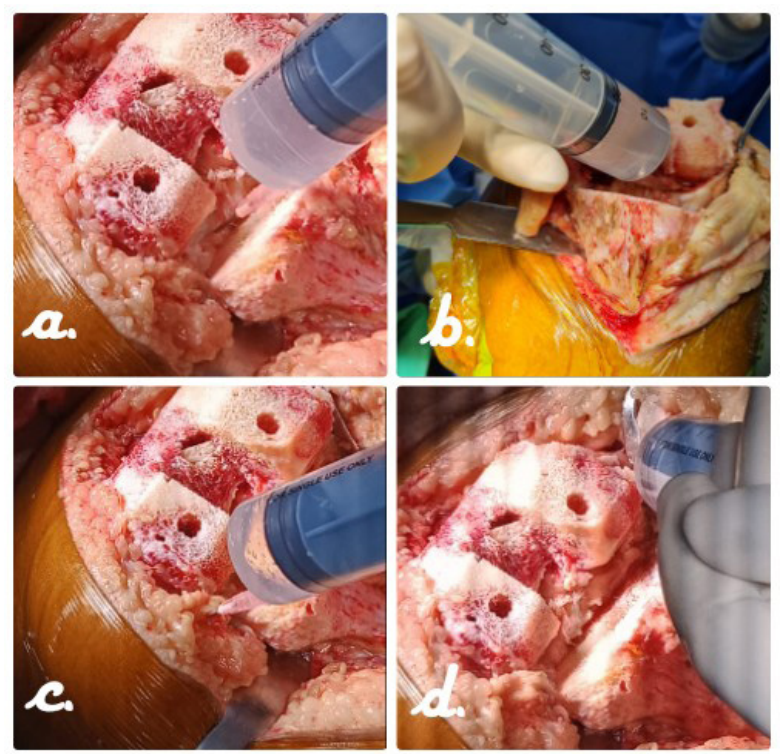

Figure 2: Periarticular Injection of "cocktail" combination of drugs in posterior capsule (a. medial side, b. lateral side) and surrounding soft tissues (c. medial side, d. lateral side) 


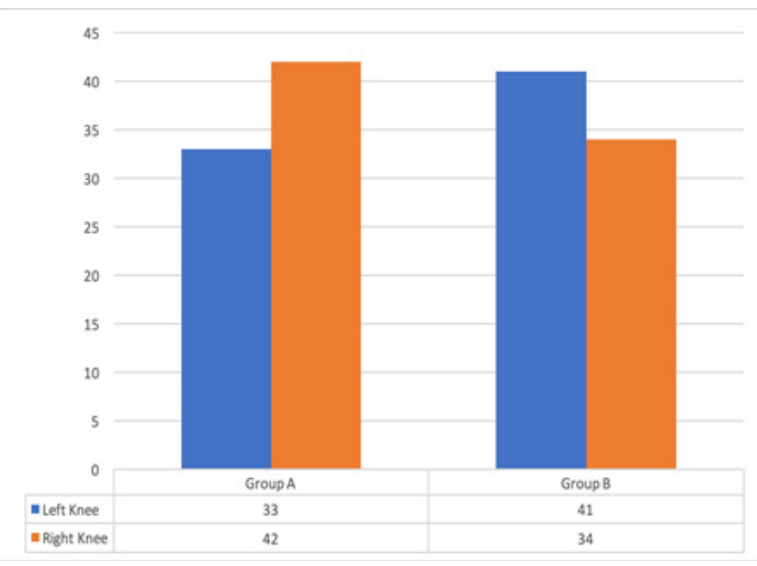

Figure 3: Frequency of knees operated in both groups

possible). Knee society score was used to evaluate the pain and function pre-operative and 3 months postoperative.

Throughout the study duration, in total 177 patients underwent TKA. Out of them, 12 patients didn't meet the inclusion criteria and 15 patients declined to participate in the study. Hence total of 150 patients were included in this study with 75 each for the Group-A and Group-B. Mean VAS score of both group was compared by using independent sample t-test and bivariate correlation was analysed using IBM SPSS software version 16.

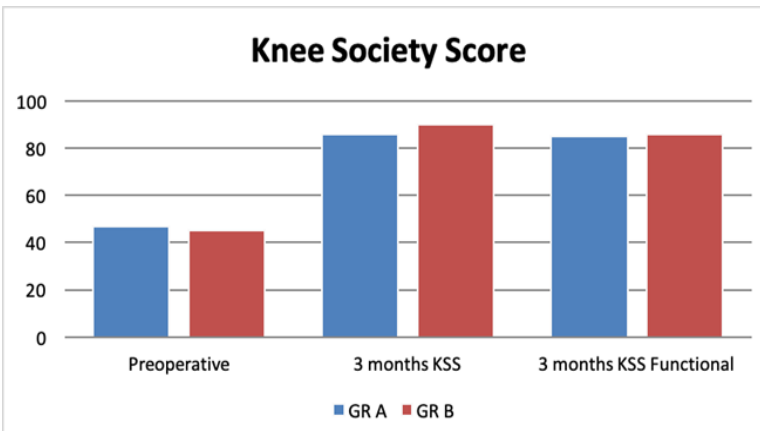

Figure 4: Functional improvement after surgery shown by Knee Society Score

\section{RESULTS}

There were 75 patients in both groups with comparable mean ages (Table 1). The Control group (group A) had 13 males and 62 females whereas the periarticular injection group (group B) had 25 males and 50 females. Females outnumbered males in both groups and overall, as well. In the control group (group A), more left knees were operated whereas more right knees were operated in the periarticular injection group i.e., Group B (Figure 3). The pain of patients was recorded at 6 hours' post-surgery (VAS0), on the 1st postoperative day (VAS1), 2nd postoperative day (VAS2), and 3rd postoperative day (VAS3) in both groups A \& B and it showed significantly better results in periarticular

Table 1: Age distribution in both groups

\begin{tabular}{lcllc}
\hline Group & Minimum Age & Maximum Age & Mean Age & Std. Deviation \\
\hline $\begin{array}{l}\text { Non-Injection } \\
\text { (Group-A) }[\mathrm{n}=75]\end{array}$ & 55.00 & 74.00 & 64.81 & 4.01 \\
$\begin{array}{l}\text { Injection Group } \\
\text { (Group-B) }[\mathrm{n}=75]\end{array}$ & 58.00 & 78.00 & 65.95 & 4.29 \\
\hline
\end{tabular}

$\$$

Table 2: Mean \& Std. deviation of VAS between both groups at $0,1,2, \& 3$ postoperative days $(n=150)$

\begin{tabular}{llllcc}
\hline VAS & $\begin{array}{l}\text { Group (each } \\
\text { with } \mathbf{n = 7 5 )}\end{array}$ & $\begin{array}{l}\text { Mean VAS- } \\
\text { score }\end{array}$ & Std. Deviation & t-value & p-value \\
\hline Day 0 & Non-Injection & 7.90 & 1.02 & 2.618 & .005 \\
& Injection & 7.52 & 0.78 & & \\
Day 1 & Non-Injection & 7.08 & 0.97 & 5.354 & $<.001$ \\
& Injection & 6.32 & 0.76 & & \\
Day 2 & Non-Injection & 6.52 & 0.98 & 10.715 & $<.001$ \\
& Injection & 5.08 & 0.63 & & \\
Day 3 & Non-Injection & 5.31 & 0.75 & 13.946 & $<.001$ \\
& Injection & 3.77 & 0.58 & & \\
\hline
\end{tabular}


injection group (Table 2). The Independent Samples t-test which is used for comparison of Means between two independent groups showed significant difference in means between the control and injection groups on each day. These differences were statistically significant as demonstrated by the P-values $(<0.05)$ on days $0,1,2 \& 3$ (Tables 2). The Knee Society Score showed functional improvement in both groups at 3 months' follow-up and only a marginal difference in functional score was seen in the injection group which wasn't statistically significant (Figure 4).

Patients of the control group demanded more postoperative additional analgesia (though both groups followed the same postoperative analgesia regime) in comparison to the periarticular injection group. Regarding postoperative complications, 1 patient from each group developed a wound infection which was managed with debridement and intravenous antibiotics. One more patient developed cellulitis from the injection group which was managed with intravenous antibiotics.

\section{DISCUSSION}

It's well established now that total knee arthroplasty has brought incredible changes in patient's life suffering from degenerative joint disease. Despite this, TKA is still an underused procedure and one major reason behind that is the fear of postoperative pain. ${ }^{1}$ Bone or soft tissue injuries during surgery, tourniquet application, and hyper-perfusion following its release are the postulated hypotheses in literature for this pain. ${ }^{11,12}$ People have different thresholds to this pain ${ }^{11}$ and as no "gold standard" regimen exists for this post-operative pain management, individual surgeons have their protocols. ${ }^{3}$ A multimodal technique named "local infiltration analgesia (LIA)" is considered safe, effective, and practical for the purpose. ${ }^{13,14} \mathrm{LIA}$ not only reduces the post-operative analgesics requirements but also reduces hospital stay. ${ }^{15}$

Different cocktail combination of medicines and different modalities of its administration has been mentioned in literature. In a study by Ranawat and Ranawat, ${ }^{1}$ they have used $0.5 \%$ bupivacaine, morphine sulfate, epinephrine, methylprednisolone acetate (excluded in diabetics and immunocompromised), cefuroxime, and normal saline as a cocktail for intraoperative injection. This cocktail injection was given before insertion of liner or doing reduction and even after the reduction. A similar protocol was used in another study by Parvataneni et al. ${ }^{2}$ In our study, we used a cocktail of only 3 drugs $(0.25 \%$ bupivacaine hydrochloride, ketorolac tromethamine, and morphine sulfate) and the cocktail was injected before insertion of implants only. No steroids or antibiotics were used in our cocktail injection. Despite variation in components of cocktail medicines, the good results shown in our study support the belief postulated by the above studies ${ }^{1,2}$ that effective pain control can be achieved by the right cocktail in the right patient.

Even the use of pre-emptive analgesia in reducing postoperative pain following TKA is mentioned in the literature. ${ }^{1,2,16}$ This prevents induction of central sensitization preempting the post-injury hypersensitivity. ${ }^{16}$ We didn't use any kind of such pre-operative analgesia in our study. Early opening of tourniquet leads to reactive hyperemia, bleeding, and subsequent washout of injected medicines lowering the effect of cocktail drugs. Klasen et al recommend a minimum of 15 minutes for release of tourniquet following periarticular infusion of drugs. ${ }^{11}$ In our study, the intraarticular cocktail injection was given just before inserting implants and with a tourniquet on. The tourniquet was released only after complete colure of skin incision and application of compression bandage which was arbitrarily more than 20 minutes since the cocktail injection.

Regarding complications, none of the patients in the injection group experienced any intraoperative serious problems following cocktail infiltration. But cases with cardiac complications following periarticular injection have been reported in the literature. $^{3}$ Postoperative wound infection was evident in both groups and was comparable.

Most of the patients were from urban areas with good accessibility of mobile phone and they made timely follow up visits. None of the patients were lost in follow up and this was one of the biggest strengths of our study. However, our study has some limitations. A relatively small sample size was one even though the sample size of 75 in one group was enough for comparative analysis. All joints undergoing TKA were with non-inflammatory pathology (degenerative joints) only. It would have been interesting to see results in TKA of inflammatory pathology (Rheumatoid joints) where synovitis leads to more pain. There even exist confounding biases like variability in patients' tolerability to pain and use of postoperative systemic analgesia.

\section{CONCLUSION}

Patients receiving periarticular infiltration of combination drugs intraoperatively had lower VAS for postoperative pain $(p<0.001)$ and this group also showed the reduced need for analgesia 
postoperatively. Hence, periarticular infiltration of the knee during Total Knee Arthroplasty is effective and safe in the management of postoperative pain.

\section{REFERENCES}

1. Ranawat AS, Ranawat CS. Pain Management and Accelerated Rehabilitation for Total Hip and Total Knee Arthroplasty. J Arthroplasty. 2007;22(7):12-5. https://doi.org/10.1016/j.arth.2007.05.040

2. Parvataneni HK, Shah VP, Howard H, Cole N, Ranawat AS, Ranawat CS. Controlling Pain After Total Hip and Knee Arthroplasty Using a Multimodal Protocol with Local Periarticular Injections. J Arthroplasty. 2007;22(6):33-8. https://doi.org/10.1016/j.arth.2007.03.034

3. Kakar P, Deshmukh U, Gagrani V, Popli G. Post-operative analgesia regime following joint replacement. Indian J Anaesth. 2011;55(3):305. http://www.ijaweb.org/text. asp?2011/55/3/305/82681

4. McCormack K, Brune K. Dissociation Between the Antinociceptive and Anti-Inflammatory Effects of the Nonsteroidal Anti-Inflammatory Drugs: A Survey of Their Analgesic Efficacy. Drugs. 1991;41(4):533-47. https://doi.org/10.2165/00003495-199141040-00003

5. Toftdahl K, Nikolajsen L, Haraldsted V, Madsen F, Tønnesen EK, Søballe K. Comparison of peri- and intraarticular analgesia with femoral nerve block after total knee arthroplasty: A randomized clinical trial. Acta Orthop. 2007;78(2):172-9. https://doi. org/10.1080/17453670710013645

6. Andersen LØ, Husted $\mathrm{H}$, Otte KS, Kristensen BB, Kehlet $\mathrm{H}$. High-volume infiltration analgesia in total knee arthroplasty: a randomized, double-blind, placebo-controlled trial: Local infiltration analgesia in knee arthroplasty. Acta Anaesthesiol Scand. 2008 17;52(10):1331-5. https://doi.org/10.1111/j.13996576.2008.01777.x

7. Busch CA, Shore BJ, Bhandari R, Ganapathy S, MacDonald SJ, Bourne RB, et al. Efficacy of Periarticular Multimodal Drug Injection in Total Knee Arthroplasty: A Randomized Trial. J Bone Jt Surg. 2006;88(5):959-63. https://doi.org/10.2106/JBJS.E.00344
8. Feldman HS. Toxicity of local anaesthetic agents. In: Rice SA, Fish KJ, editors. Anesthetic toxicity. New York: Raven Press; 1994. p 107-33. In. [Book Section]

9. Stein C. Peripheral Mechanisms of Opioid Analgesia. In: Herz A, Akil H, Simon EJ, editors. Opioids II [Internet]. Berlin, Heidelberg: Springer Berlin Heidelberg; 1993 [cited 2020 Nov 28]. p. 91-103. (Handbook of Experimental Pharmacology; vol. 104 / 2). Available from: http://link.springer.com/10.1007/978-3-64277540-6_4

10. Scuderi GR. Peri-Articular Regional Analgesia in Total Knee Arthroplasty. A Review of The Neuroanatomy and Injection Technique. 2014;6. https://doi.org

11. Klasen JA, Opitz SA, Melzer C, Thiel A, Hempelmann G. Intraarticular, epidural, and intravenous analgesia after total knee arthroplasty: Analgesia after total knee arthroplasty. Acta Anaesthesiol Scand. 1999;43(10):1021-6. https://doi.org/10.1034/j.13996576.1999.431009.x

12. Smith I, Van Hemelrijck J, White PF, Shively R. Effects of local anesthesia on recovery after outpatient arthroscopy. Anesth Analg. 1991;73(5):536-9.

13. Kerr DR, Kohan L. Local infiltration analgesia: a technique for the control of acute postoperative pain following knee and hip surgery: A case study of 325 patients. Acta Orthop. 2008;79(2):174-83. https://doi. org/10.1080/17453670710014950

14. Horlocker TT, Kopp SL, Pagnano MW, Hebl JR. Analgesia for Total Hip and Knee Arthroplasty: A Multimodal Pathway Featuring Peripheral Nerve Block: J Am Acad Orthop Surg. 2006;14(3):126-35. https:// doi.org/10.5435/00124635-200603000-00003

15. Stein C. The Control of Pain in Peripheral Tissue by Opioids. Epstein $\mathrm{FH}$, editor. $\mathrm{N}$ Engl J Med. 1995;332(25):1685-90. https://doi.org/10.1056/ NEJM199506223322506

16. Woolf CJ, Chong MS. Preemptive analgesia - treating postoperative pain by preventing the establishment of central sensitization. Anesth Analg. 1993;77:362-79. https://doi.org/10.1213/00000539-199377020-00026 